\title{
In situ Scanning Transmission Electron Microscopy with Atomic Resolution under Atmospheric Pressure
}

\section{Sheng Dai, ${ }^{1 *}$ Shuyi Zhang, ${ }^{1}$ George W. Graham, ${ }^{1}$ and Xiaoqing Pan ${ }^{1,2}$}

${ }^{1}$ Department of Materials Science and Engineering, 644 Engineering Tower, University of California - Irvine, Irvine, CA 92697

${ }^{2}$ Department of Physics and Astronomy, University of California - Irvine, Irvine, CA 92697

*dais04thu@gmail.com

\begin{abstract}
Significant developments in micro-electrical-mechanical systems (MEMS)-based devices have led to the commercialization of windowed gas cells that now enable atomic-resolution scanning transmission electron microscopy (STEM) observation of phenomena occurring during gas-solid interactions at atmospheric pressure. An in situ atmospheric STEM study provides information that is beneficial to correlating the structure-properties relationship of catalytic nanomaterials, particularly under realistic gaseous reaction conditions. In this article, we illustrate the advantages of this tool as applied to our study of two important systems: (1) the CO-induced Pt nanoparticle surface reconstruction at saturation coverage and (2) the ordering and Pt surface enrichment in supported $\mathrm{Pt}_{3} \mathrm{Co}$ nanoparticles.
\end{abstract}

Keywords: in situ electron microscopy, windowed gas cell, atmospheric pressure, gas-solid interaction, catalytic nanomaterials

\section{Introduction}

In situ electron microscopy studies under gaseous environments have attracted attention not only for basic scientific research, but also for industrial applications. In the past decade, the majority of in situ TEM studies involving gas-solid interaction were performed in the differentially pumped environmental TEM (ETEM), which incorporates a series of differential pumping apertures and additional pumping capability to create a gaseous environment inside the microscope $[1,2]$. However, because of the current design of the ETEM, some constraints still exist: (1) the maximum gas pressure allowed in ETEM is only 15 Torr $(0.02 \mathrm{~atm})$, far from atmospheric pressure, which is not favorable for building a bridge between in situ results and real applications, particularly in catalysis research [3]; (2) applicability of atomic-resolution high-angle annular dark-field scanning transmission electron microscopy (HAADF-STEM) imaging is limited by post-specimen differential pumping apertures [4].

Recently it has become possible to overcome these limitations for in situ atmospheric scanning transmission electron microscopy (STEM) studies through the use of a micro-electrical-mechanical systems (MEMS)-based, electron-transparent windowed gas cell [5]. Using this instrumentation, the sealed gaseous environment can reach atmospheric pressures during full HAADF-STEM imaging in a state-of-art probe-corrected TEM. In this article we illustrate the advantages of the windowed gas cell technique as applied to our in situ study of two important phenomena: (1) CO-induced Pt nanoparticle surface reconstruction at saturation coverage and (2) oxygen-driven $\mathrm{Pt}$ surface enrichment on supported $\mathrm{Pt}_{3} \mathrm{Co}$ nanoparticles.

\section{Materials and Methods}

The design intent of the windowed gas cell is to modify the TEM specimen holder by placing a pair of electron transparent "windows" above and below the specimen to seal the gas atmosphere from the high vacuum of the TEM column (Protochips Atmosphere system), as shown in Figures 1a and $1 \mathrm{~b}$. Gas inlet and outlet tubes run through the specimen holder rod. The windows are made of amorphous $\mathrm{SiN}_{\mathrm{x}}$ to take advantage of this material's elastic properties and high fracture strength. These mechanical properties make the $\mathrm{SiN}_{\mathrm{x}}$ amorphous membrane strong enough to confine a pressurized gas up to four atmospheres [6]. In addition, the low electron diffraction contrast of the amorphous $\operatorname{SiN}_{\mathrm{x}}$ membrane does not limit the superposition of signals coming from the sample.

The gas cell holder can be safely inserted in conventional TEMs without modification to the column or vacuum system, which results in direct savings in terms of instrument cost and maintenance. No differential pumping apertures are required in this cell set up, making the electrons scattered at high angles available to the detectors and removing some of the limitations

(a)

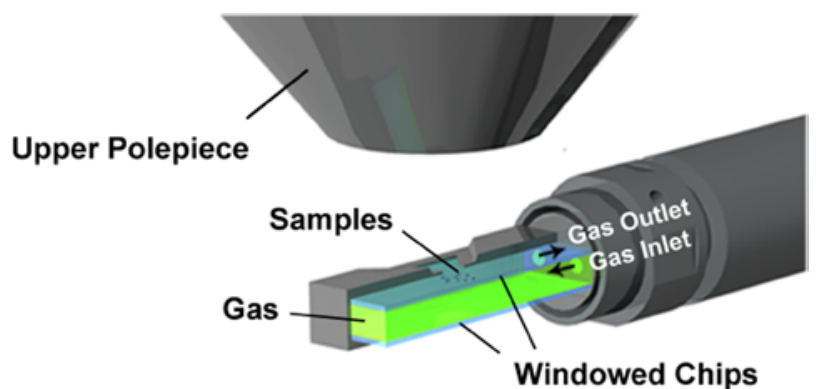

Lower Polepiece

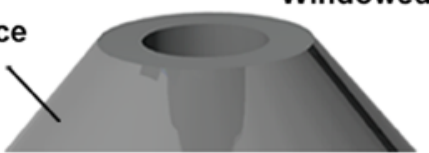

(b)

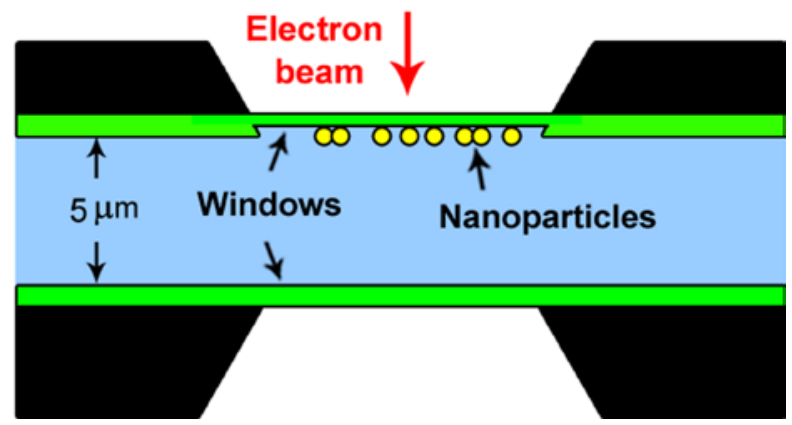

Figure 1: Schematic representation of the windowed gas cell TEM holder for in situ atmospheric STEM. (a) Cut-away view of windowed gas cell in the microscope gap (not to scale). (b) Cross-section drawing illustrating the structure of windowed gas cell. The black region is spacer, the green window is $\mathrm{SiN}_{\mathrm{x}}$, and the light blue region indicates the gaseous area. 


\section{YOU'LL FIND DATOOMEAT THE FOREFRONT OF TWOLATION.}
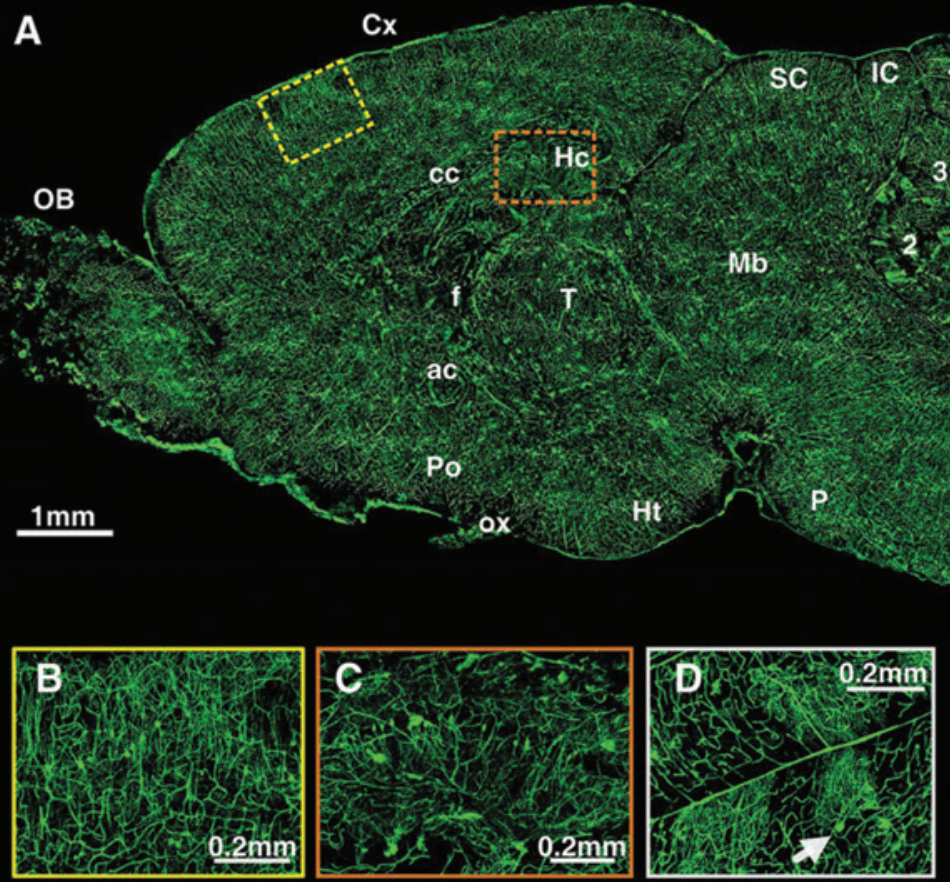

Sk

\section{Greating a High Resolution Atlas of the Mouse Brain...}

(A) A sagittal image reconstructed from a stack of 100 virtual sagittal sections (total thickness of $0.1 \mathrm{~mm}$ ). These sections were transformed from the original coronal sections. The sagittal image was located in the right hemisphere about $0.4 \mathrm{~mm}$ lateral to the middle. Almost all major regions of the brain can be seen in this image, e.g., the Olfactory Bulb (OB), Cerebral Cortex (CX), Hippocampus (Hc), Fornix(f), Anterior Commissure (ac), Thalamus (T), Cerebellum (Cb), Midbrain (Mb), Pons (P), Medulla (Md), Corpus Callosum (cC), Superior Colliculus (SC), Inferior Colliculus (IC), Hypothalamus (Ht), Preoptic Area (Po), Optic Chiasm (ox), 4 th ventricle (4V) and nine lobules of the cerebellum (Arabic numerals, 2 to 10). The three regions inside the different colored rectangle in (A) are the positions of (B), (C) and (D), which illustrate the cerebral cortex, hippocampus and cerebellum, respectively. In the reconstruction of sagittal image, no dislocation was observed along the D-V axis, i.e., the coronal sections are inherently aligned along the A-P axis.

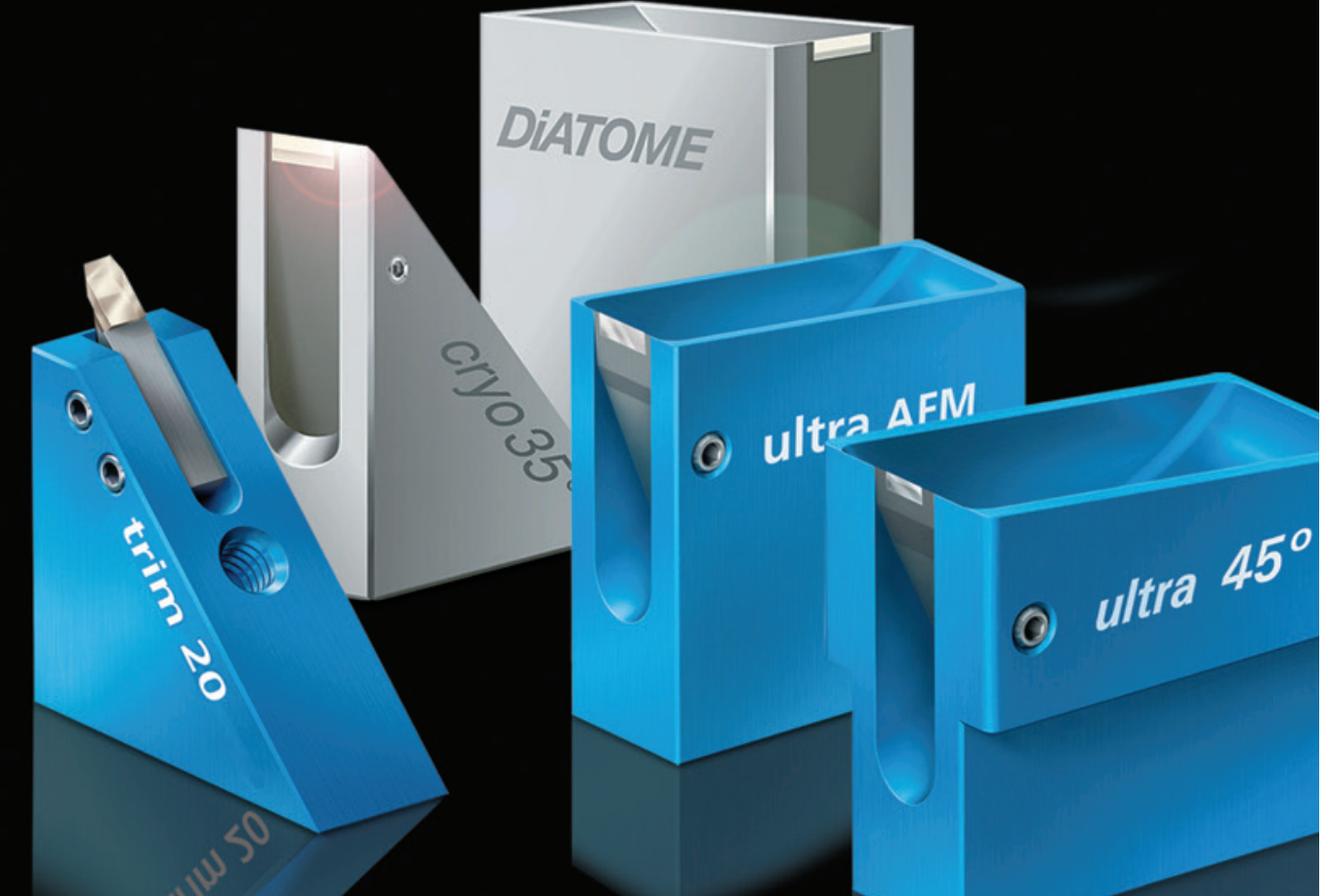

\section{DIATOME QUALITY AND INNOVATION APPLIED..}

Micro-Optical Sectioning Tomography to Obtain a High-Resolution Atlas of the Mouse Brain

Existing imaging tools have limitations for brainwide mapping of neural circuits at a mesoscale level. In collaboration with DiATOME, researchers developed a Micro-Optical Sectioning Tomography (MOST) system utilizing a DiATOME Diamond Knife that can provide micron tomography of a centimeter-sized whole mouse brain.

Slicing was performed by moving the specimen to generate ribbons, and each ribbon was simultaneously imaged. The illuminating beam passed through a beam splitter, mirror and objective to irradiate the ribbon. The imaging beam collected by the objective and passed through the mirror, beam splitter and tube lens was then recorded by a line-scan CCD.

A 3D structural dataset of a Golgi-stained whole mouse brain at the neurite level was obtained. The morphology and spatial locations of neurons and traces of neurites were clearly distinguished. Researchers found that neighboring Purkinje cells were sticking to each other.

\section{Acknowledgement}

Micro-Optical Sectioning Tomography to Obtain a High-Resolution Atlas of the Mouse Brain Anan Li, Hui Gong, Bin Zhang, Qingdi Wang. Cheng Yan, Jingpeng Wu, Qian Liu, Shaoqun Zeng, Qingming Luo Britton Chance Center for Biomedical Photonics, Wuhan National Laboratory for Optoelectronics-Huazhong University of Science and Technology, Wuhan 430074, P. R. China.

ACHIEVE PUITING EDGE RESULTS WITH DATOME DMMOND KNIVES

\section{PLEASE CONTACT US FOR MORE INFORMATION...}

\section{DIATOME U.S.}

P.0. Box $550 \bullet 1560$ Industry Rd.

Hatfield, PA 19440

Tel: (215) 412-8390 • Fax: (215) 412-8450

email: info@emsdiasum.com

orstacie@ems-secure.com

\section{www.emsdiasum.com}

follow us on...

$f$ in $g_{+} \circ$ 의



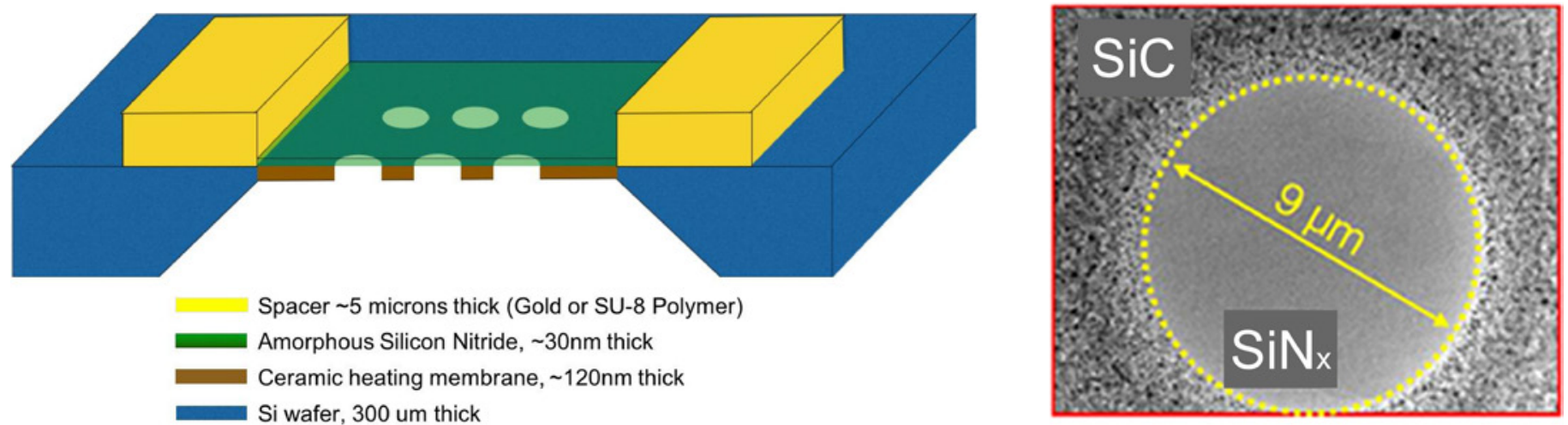

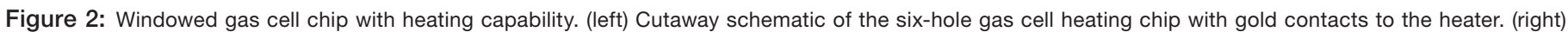
Bright-field image of one of the holes in the $\mathrm{SiC}$ ceramic and the $\mathrm{SiN}_{\mathrm{x}}$ membrane window.

of HAADF-STEM imaging encountered in the differentially pumped ETEM. In addition, most powder samples can be easily loaded on this gas cell holder. For example, the powders are first dispersed in solvent, and the suspension is deposited directly onto one of the windows (the upper one or the lower one, depending on whether TEM mode or STEM mode is to be used). The powder samples are thus situated between the two windows, sealed in a gaseous environment.

Meanwhile, MEMS-based technology enables the integration of the $\mathrm{SiN}_{\mathrm{x}}$ windows into $\mathrm{Si}$ chips with heating capabilities. As shown in the schematic of the Protochips Atmosphere gas cell system (Figure 2), both chips are etched in the center region and support a thin $(<50 \mathrm{~nm})$ amorphous $\mathrm{SiN}_{\mathrm{x}}$ window over the open imaging area. In one style of the window chip, the amorphous $\mathrm{SiN}_{\mathrm{x}}$ window is supported over an array of 9 $\mu \mathrm{m}$ holes patterned in a thin low-conductivity $\mathrm{SiC}$ ceramic (see cutaway schematic in Figure 2), which serves as a heating unit. Gold electrode contact pads are fabricated on the two chips to allow current to pass from electrical leads coming through the specimen holder. With this design the heating membrane can achieve heating and cooling rates on the order of $10^{6}{ }^{\circ} \mathrm{C} / \mathrm{s}$, allowing more rapid thermal response and stabilization than with standard heating holders. It has been demonstrated that atomic resolution can be realized even at $1000{ }^{\circ} \mathrm{C}$ and $1 \mathrm{~atm}$ pressure in an aberration-corrected STEM/TEM instrument [7].

\section{Results}

CO-induced Pt nanoparticle surface reconstruction This example is an observation of structural rearrangement on the surface of a $\mathrm{Pt}$ nanoparticle induced by adsorption of $\mathrm{CO}$ at saturation coverage and elevated temperature [8]. It is well known that the adsorption of reactive gaseous species can cause the reconstruction of metal surfaces, and it is also known that different configurations of surface atoms have different catalytic reactivities [9]. Thus, characterizing metallic surface structures under gaseous reaction conditions at atomic scale is critical for understanding reactivity.

(c)
The specimen consists of $\sim 10 \mathrm{~nm}$ diameter Pt nanoparticles in the shape of truncated octahedrons supported on carbon. As illustrated in Figure 3a, the atomic-scale HAADFSTEM image, taken at $150^{\circ} \mathrm{C}$ in 500 Torr of $\mathrm{N}_{2}$, shows an edge view of flat $\{100\}$ atomic planes at the surface of the as-prepared Pt nanoparticle (beam along the $<110>$ zone axis). This $\{100\}$ surface configuration was stable under $\mathrm{N}_{2}$ atmosphere at $150{ }^{\circ} \mathrm{C}$, and no morphological changes were identified during 30 minutes of in situ observation. At the end of that initial observation, and while the $150{ }^{\circ} \mathrm{C}$ temperature was maintained, the $\mathrm{N}_{2}$ was pumped out, and 500 Torr of reactive gas (5 vol\% CO in $95 \% \mathrm{Ar}$ ) was introduced into the gas cell. It is notable that the partial pressure of $\mathrm{CO}$ reached 25 Torr, ensuring a saturation CO coverage on the Pt nanoparticle surfaces (saturation requires $\sim 10$ Torr) [10]. As a result, Figure $3 \mathrm{~b}$ shows an in situ HAADF-STEM image of the same Pt nanoparticle after exposure to the $\mathrm{CO}$ environment for 10 minutes. For a clear comparison of structural differences induced by $\mathrm{CO}$ exposure, layers 1 and 3 are marked as a reference in both the initial and reconstructed states. Upon $\mathrm{CO}$ adsorption, a new atomic plane of $\mathrm{Pt}$ atoms (marked as Layer 0) emerged on top of the original
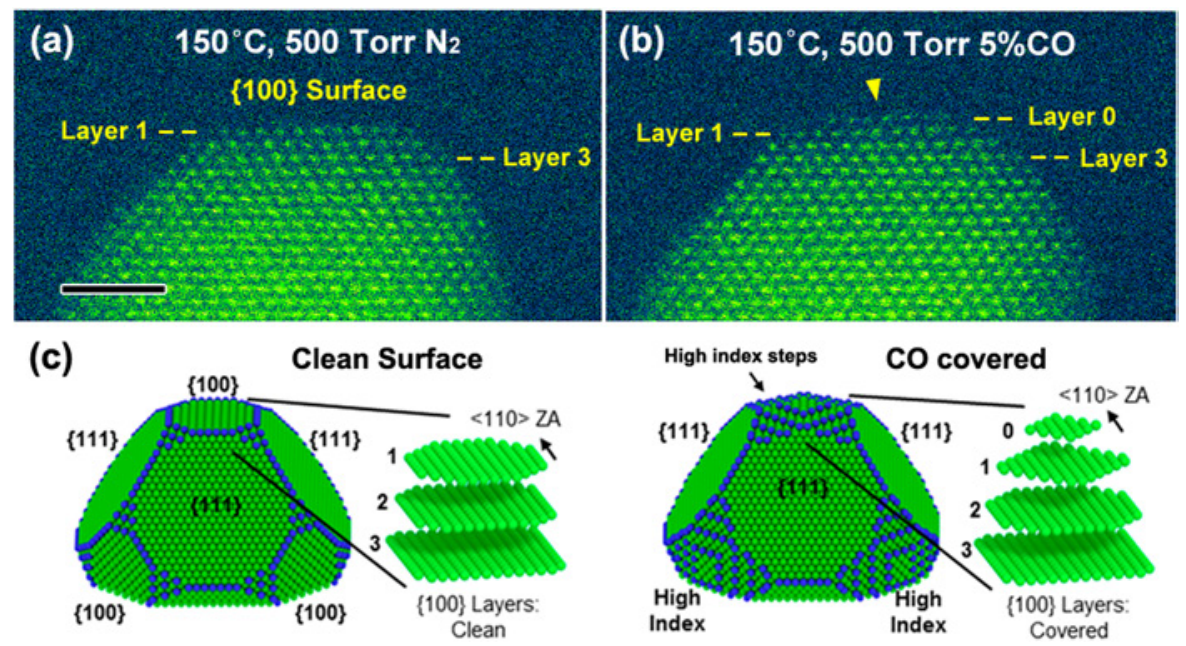

Figure 3: In situ STEM measurement of CO-induced Pt nanoparticle surface reconstruction. (a) HAADFSTEM image of a Pt nanoparticle under a $\mathrm{N}_{2}$ environment. Scale bar $=2 \mathrm{~nm}$. (b) Same particle under a $\mathrm{CO}$ environment, showing a surface reconstruction on the $\{100\}$ surface. (c) Wulff constructions of a $\mathrm{Pt}$ nanoparticle, based on DFT-calculated surface-free energies of clean surfaces and CO-saturated surfaces. 
$\{100\}$ surface, indicated by the yellow arrow in the experimental image (Figure 3b).

A density functional theory (DFT) calculation based on Wulff construction models was performed by using the real space grid-based projector-augmented wave method (GPAW) open source code [11]. The surface energies of bare Pt facets and CO-covered Pt facets were calculated. After CO adsorption, the surface free energy of the $\{100\}$ facet was comparable to that of stepped vicinal surfaces, for example, $\{210\}$ and $\{310\}$, which are higher index surfaces likely to be more reactive. Thus, as shown in Figure 3c, the newly formed Layer 0 is created through the migration of $\mathrm{Pt}$ atoms from lower $\{100\}$ layers (for example, Layer 1 and Layer 2). More under-coordinated Pt surface atoms (with metal-metal coordination numbers of 6 or 7 ), as indicated by blue atoms in Figure 3c, come into appearance next to the (100) surface. Both the STEM image and the DFT simulations suggest that under saturation CO coverage, the terminating Pt $\{100\}$ facets will reconstruct to vicinal steps. Upon further $\mathrm{CO}$ annealing at a higher temperature of $300{ }^{\circ} \mathrm{C}$ for 10 minutes, the layer 0 was observed to disappear and the $\mathrm{Pt}$ particle reverted to its original shape [8]. The reversibility of the $\mathrm{Pt}$ surface reconstruction reflects the desorption of $\mathrm{CO}$ molecules, shown by in situ infrared spectroscopy to take place above $\sim 230{ }^{\circ} \mathrm{C}[12]$.

Ordering and $\mathrm{Pt}$ surface enrichment in $\mathrm{Pt}_{3}$ Co nanoparticles The second example we present is the in situ STEM observation of surface evolution in ordered $\mathrm{Pt}_{3} \mathrm{Co}$ nanoparticles [13], which is important for the oxygen reduction reaction (ORR) in fuel cell catalysts. To better understand the formation process of a Pt shell, which is crucial for enhancing ORR activity, we performed an in situ experiment on carbon-supported $\mathrm{Pt}_{3} \mathrm{Co}$ nanoparticles under static pure oxygen at 760 Torr.

During the in situ experiment, the temperature was first elevated to $720{ }^{\circ} \mathrm{C}$, which is close but below to the orderdisorder transition temperature of a 75 at\%Pt-25 at\%Co alloy, at a high rate of $5{ }^{\circ} \mathrm{C} / \mathrm{s}$. Upon oxygen annealing at $720{ }^{\circ} \mathrm{C}$ for 30 min, the initially disordered $\mathrm{Pt}_{3} \mathrm{Co}$ nanoparticles were transformed to an ordered intermetallic $\mathrm{L}_{2}$ phase where Pt surface segregation had also taken place to form a two-layer Pt-rich shell. Away from the particle surface, the ordered (100) planes alternate as pure $\mathrm{Pt}$ and pure Co planes. In the atomic-level HAADF-STEM imaging of the (100) planes, the last Co layer at the particle edge (low intensity) provided a marker and was labeled the \#0 layer. Additional Pt layers caused by the processing in oxygen then could be identified and counted as layers \#1 through \#4.

After the processing above, the temperature was rapidly cooled to $300{ }^{\circ} \mathrm{C}$. The surface configuration of the $\mathrm{Pt}_{3} \mathrm{Co}$ nanoparticle was stable as shown in Figures $4 \mathrm{a}$ and $4 \mathrm{~d}$. Sequential atomic-scale HAADF-STEM images were then taken at a scanning speed at one frame every $16 \mathrm{~s}$ at $300{ }^{\circ} \mathrm{C}$. As shown in Figure $4 \mathrm{~b}$, the $\mathrm{Pt}_{3} \mathrm{Co}$ nanoparticle was found to be larger at an elapsed time of $64 \mathrm{~s}$ with the measured diameter, $d_{64 \mathrm{~s}}$, of $13.18 \mathrm{~nm}$, showing an increase of $\Delta d=0.39 \mathrm{~nm}(3.90$
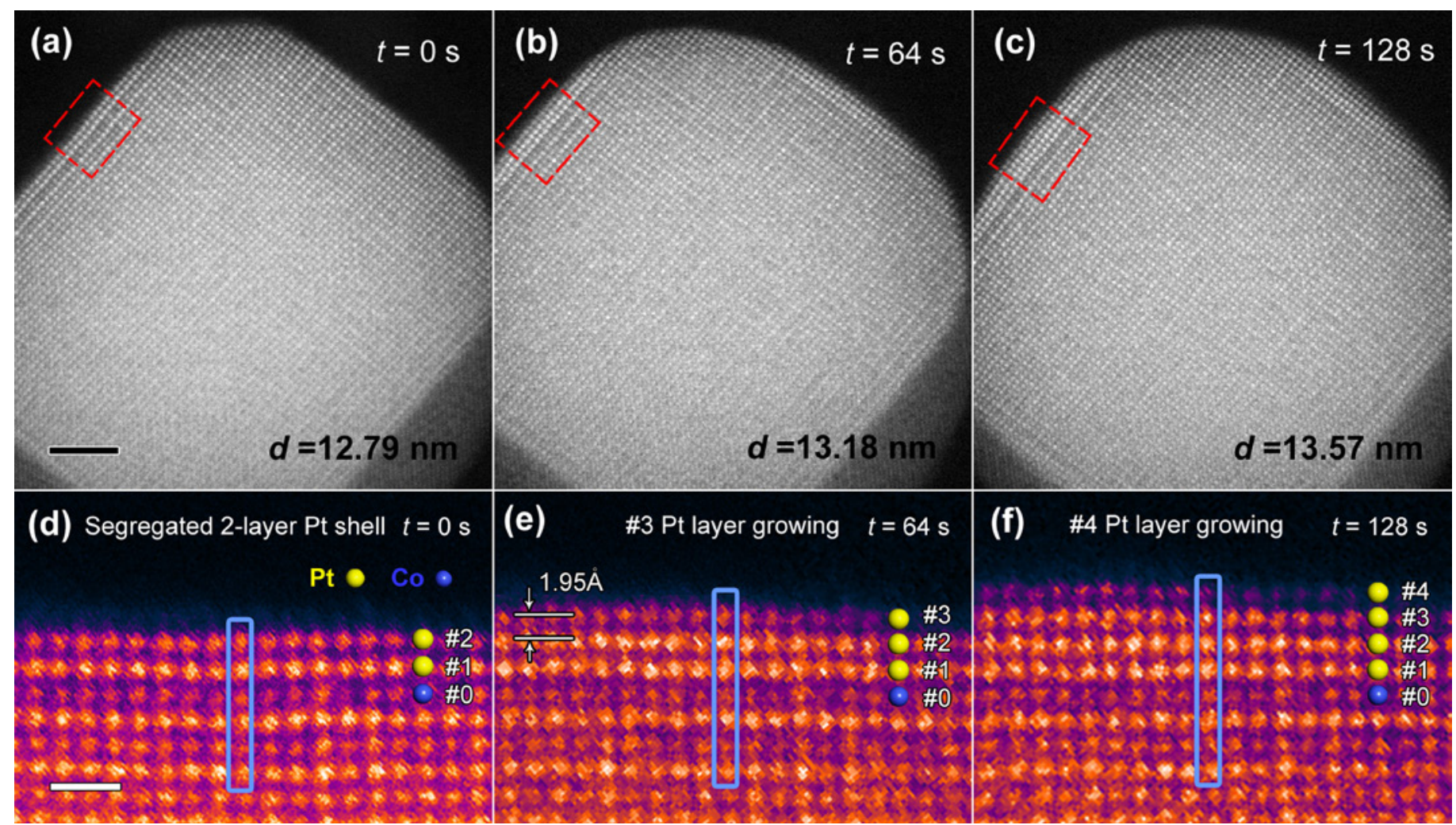

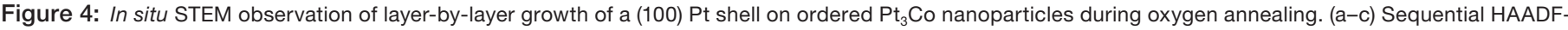

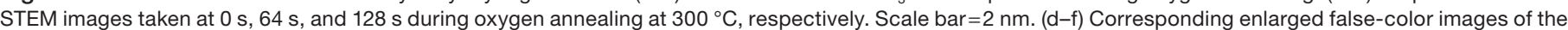

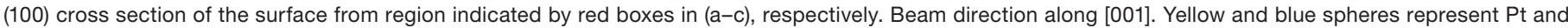
Co atoms, respectively. Scale bar $=0.5 \mathrm{~nm}(5 \AA)$. 
$\AA$ ), compared to the particle status in Figure 4a. In the corresponding enlarged panel, Figure 4e, it is evident that an extra atomic layer (layer \#3) had grown on the (100) surface. The lattice spacing between layer \#2 and layer \#3 is found to be 0.195 $\mathrm{nm}(1.95 \AA)$, which is exactly half of $\Delta d$. This indicates another extra atomic layer has also started growing on the opposite (100) facet at the lower-right corner of Figure $4 \mathrm{~b}$. Similarly, by comparing Figures $4 \mathrm{a}$ and $4 \mathrm{~b}$, an extra layer can also be identified on the other two (100) surfaces (the one at the top right and the one at lower left). Furthermore, at an elapsed time of $128 \mathrm{~s}$, Figure $4 \mathrm{c}$, the measured diameter, $d_{128 \mathrm{~s}}=13.57 \mathrm{~nm}$, indicating a further increase of $0.39 \mathrm{~nm}(3.90 \AA)$, demonstrating that the particle continued to undergo layer-by-layer growth at (100) surfaces. The atomic resolution image of Figure $4 \mathrm{f}$ shows the newly grown layer \#4.

To determine the composition of the newly grown layers, HAADF-STEM intensity analysis was performed by using Gatan Microscopy Suite Software. Intensity profiles were obtained from the same (020) plane indicated by blue boxes in Figures $4 \mathrm{~d}-4 \mathrm{f}$ at $\mathrm{t}=0,64$, and $128 \mathrm{~s}$, respectively. Then, as summarized in Figure 5, the new layers \#3 and \#4 can be quantitatively represented in the sequential intensity profiles. Comparison of profiles (ii) and (iii) indicates that the intensity of layer \#3 is increasing during the interval of $64 \mathrm{~s}-128 \mathrm{~s}$. At 128 $\mathrm{s}$, layer \#3 may be considered completely grown since the new layer \#4 has started growing. The columns in layer \#3 exhibit an intensity similar to that of the segregated Pt layers \#1 and \#2, which is higher than that of the inner Co columns. For confirmation, a HAADF-STEM image simulation was performed based on a segregated intermetallic $\mathrm{Pt}_{3} \mathrm{Co}$ model with a threelayer pure Pt shell, by using the QSTEM simulation package [14]. According to the simulation results, the intensity profile of the three-layer Pt shell shows the same trend as the corresponding layers \#1-\#3 in the experimental profile (iii), supporting the experimental result that the newly grown layer is, indeed, a Pt-rich shell.

\section{Discussion}

Using a windowed gas cell specimen holder for in situ STEM, we observed surface phenomena that are difficult or impossible to observe by other methods. Direct observation of the reconstruction of metallic surfaces at realistic temperatures and pressures has been sought for decades.

In the first experiment, the truncated octahedron shape adopted by bare Pt nanoparticles was observed to reconstruct as a result of $\mathrm{CO}$ adsorption. In this facet-specific process, flat (100) facets roughen into vicinal stepped high Miller index facets, while it is assumed that flat (111) facets remained intact. The relatively high partial pressure of $\mathrm{CO}(>20$ Torr), allowed in the windowed gas cell, ensured saturation $\mathrm{CO}$ coverage, which triggered the observed surface reconstruction of $\mathrm{Pt}$ nanoparticles. (i) $t=0 \mathrm{~s}$

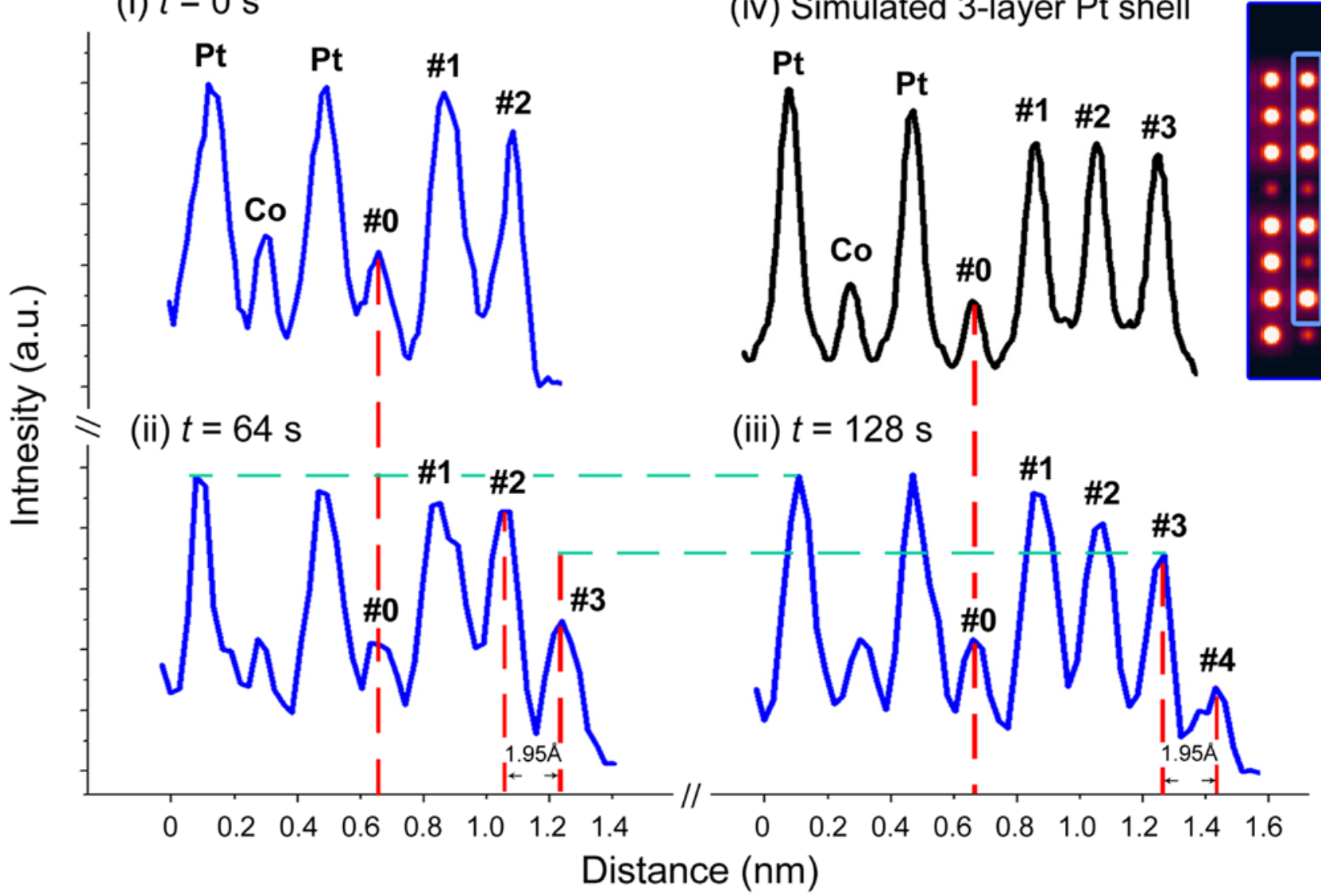

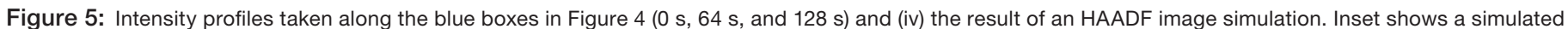
image of the three-layer Pt shell on the (100) surface. 
In the second experiment, the alternating Pt and Co (100) planes of ordered $\mathrm{Pt}_{3} \mathrm{Co}$ particle provided an internal marker for observation of surface reconstruction in oxygen. This allowed atomic-level compositional imaging at the surface. The details of this observed oxygen-driven Pt surface enrichment may pave a way for designing and tailoring the structure and performance of the Pt-Co ORR catalyst. This case study highlights the capability of Z-contrast HAADF-STEM imaging combined with image simulation for analysis of chemical composition changes at nanoparticle surfaces under realistic catalytic conditions.

Thus, a MEMs-based window gas cell enables an in situ atmospheric STEM study at atomic resolution to explore novel and interesting phenomena occurring during gas-solid interactions. However, some limitations still exist in the current form of the windowed gas cell for more general analyses on a wider range of materials. These limitations include a lower contrast for light-element materials (for example, graphene, boron nitride) because of the dimension of the $\mathrm{SiN}_{\mathrm{x}}$ windows and the single-tilting function of the current design. But it may only be a matter of time before these constraints are removed. We believe that atomic-resolution in situ STEM under atmospheric pressure will be an essential tool for advanced research of gas-solid interactions, and numerous exciting findings can be expected.

\section{Conclusion}

In summary, the windowed gas cell now allows atomicresolution STEM observation of dynamic structure evolution of catalytic nanomaterials under realistic gaseous conditions, providing information that is beneficial to understanding their structure-properties relationship. The two application examples presented here clearly demonstrate the advantages and capability of this in situ technique, particularly the high spatial resolution and high stability under atmospheric gas pressure.

\section{Acknowledgments}

The authors thank Protochips Inc. for the technical support of the Atmosphere windowed gas cell facility. Additional support was provided by the University of California - Irvine Materials Research Institute (IMRI) for use of the state-of-theart TEMs.

\section{References}

[1] R Sharma, J Mater Res 20 (2005) 1695-1707.

[2] ED Boyes, Ultramicroscopy 67 (1997) 219-32.

[3] Y Li , Nat Commun 6 (2015) 7583.

[4] JR Jinschek, Chem Commun 50 (2014) 2696-06.

[5] N de Jonge, Nano Lett 10 (2010) 1028-32.

[6] T Alan, Appl Phys Lett 100 (2012) 081903.

[7] LF Allard, Microsc Microanal 18 (2012) 656-66.

[8] T Avanesian, J Am Chem Soc 139 (2017) 4551-58.

[9] J Gu, Chem Soc Rev 41 (2012) 8050-65.

[10] SR Longwitz, J Phys Chem B 108 (2004) 14497-14502.

[11] J Enkovaara , J Phys Condens Matter 22 (2010) 253202.

[12] MJ Kale, ACS Catal 6 (2016) 5599-5609.

[13] S Dai , Nat Commun 8 (2017) 204.

[14] CT Koch. Ph.D. Thesis, Arizona State University, 2002.

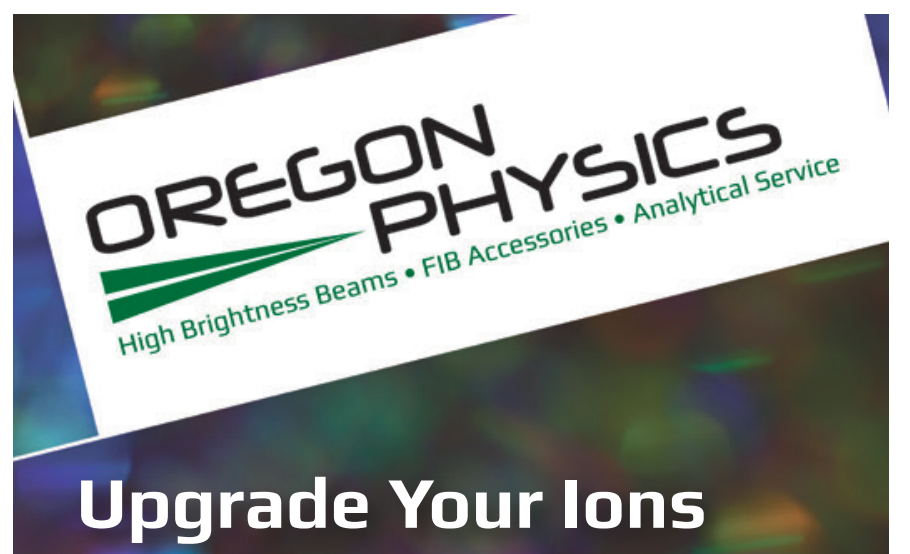

Hyperion ${ }^{\text {TM }}$ Dual Polarity Ion Sources are now available as direct upgrades from Oregon Physics for FEI FIB 200, PHI Adept 1010, and Cameca NanoSIMS, IMS F series, and 12XX series instruments.

Upgrade your ion source to benefit from:

- Longer source lifetime

- Better image resolution

- Improved depth profiling (SIMS)

- Higher currents for milling (FIB)

Oregon Physics' Hyperion ion sources are designed to bolt-on to your existing optical system for easy implementation.

How will Hyperion improve your research? Learn more at Oregon-Physics.com or call us to discuss your requirements.

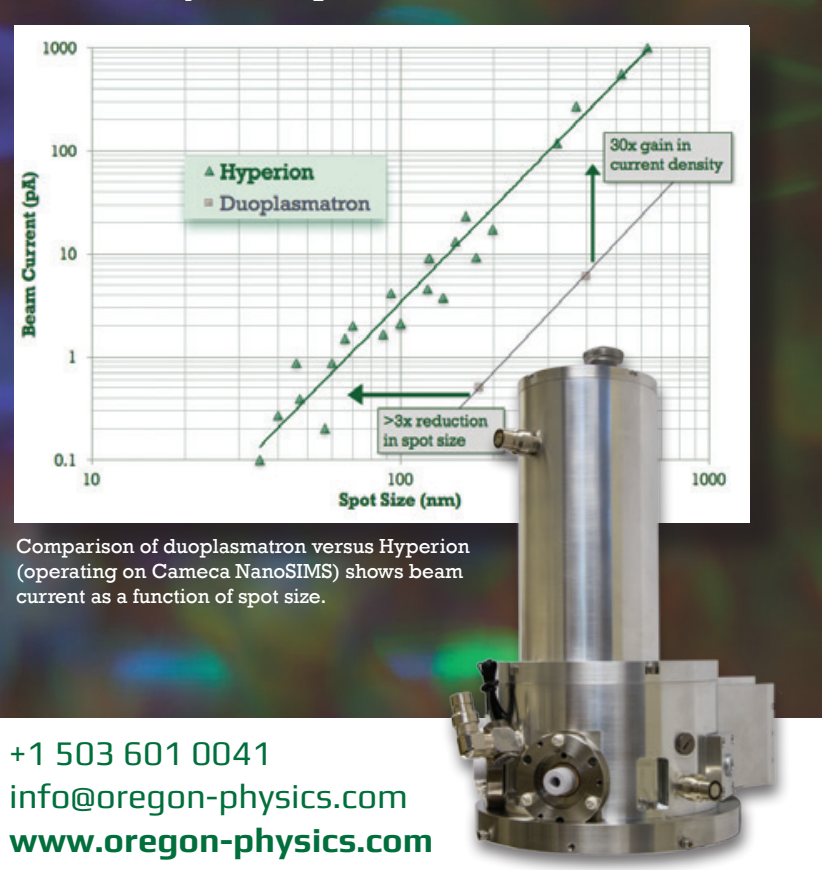

